\title{
Occurrence and distribution of antibiotic resistance genes in sediments in a semi-enclosed continental shelf sea
}

\author{
Yuxuan Zhang ${ }^{\mathrm{a}, \mathrm{b}}$, Jian $\mathrm{Lu}^{\mathrm{a}, \mathrm{b}, \mathrm{c}, *}$, Jun $\mathrm{Wu}^{\mathrm{d}}$, Jianhua Wang ${ }^{\mathrm{a}}$, Yichen Lin ${ }^{\mathrm{a}, \mathrm{b}}$

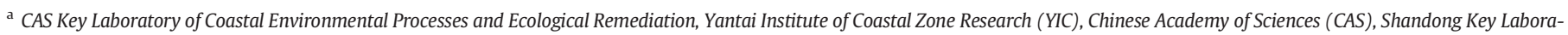 \\ tory of Coastal Environmental Processes, YICCAS, Yantai, Shandong 264003, PR China \\ ${ }^{\mathrm{b}}$ University of Chinese Academy of Sciences, Beijing 100049, PR China \\ c Center for Ocean Mega-Science, Chinese Academy of Sciences, 7 Nanhai Road, Qingdao 266071, PR China \\ d School of Resources and Environmental Engineering, Ludong University, Yantai, Shandong 264025, PR China
}

\section{H I G H L I G H T S}

- Pollution of ARGs in sediments from the Bohai Sea areas was firstly investigated.

- ARGs abundances varied over 3 orders of magnitude in different sediments.

- ARGs were more abundant in the Laizhou Bay and eastern of central sea basin.

- Abundance of sulfonamides resistance genes ranged from $5.7 \times 10^{4}$ to $1.8 \times 10^{7}$ copies $/ g$.

- Potential host bacteria of ARGs were Lysobacter, Thiobacillus, and Defluviimonas.

\section{A R T I C L E I N F O}

\section{Article history:}

Received 29 November 2019

Received in revised form 26 February 2020

Accepted 2 March 2020

Available online 3 March 2020

\section{Keywords:}

Antibiotic resistance genes

Semi-enclosed continental shelf sea

Spatial distribution

Potential host bacteria

Bacterial communities

Network analysis
GRAPHICALA B STRACT

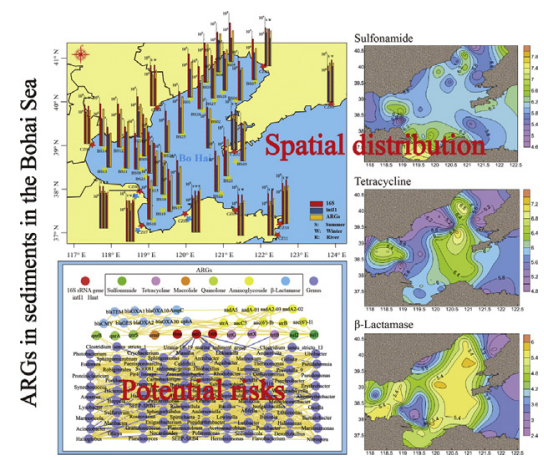

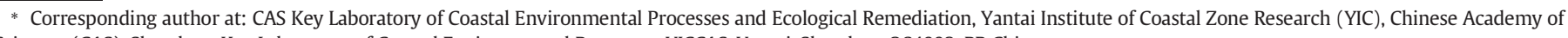
Sciences (CAS), Shandong Key Laboratory of Coastal Environmental Processes, YICCAS, Yantai, Shandong 264003, PR China.

E-mail address: jlu@yic.ac.cn (J. Lu).
} 
possible hosts of ARGs were revealed by network analysis with higher relative abundance in coastal areas than the sea.

\section{Introduction}

Antibiotics have been widely used as medicines for the cure of human and livestock diseases, and the growth promoters in animal husbandry. However, extensive and improper use of antibiotics has led to the prevalence of antibiotic resistance (Zhang et al., 2015). A lot of investigations focused on the occurrence, propagation and dissemination of antibiotic resistance genes (ARGs) in wastewater and sludge in wastewater treatment plants (Lu et al., 2020a, 2020b; Yin et al., 2019), livestock breeding (Jia et al., 2017), aquacultural systems (Wang et al., 2018) and landfill (Zhao et al., 2018), or water and sediments in rivers (Dang et al., 2017), estuaries (Zhu et al., 2017) and public parks (Wang et al., 2014), etc. All of these targets, especially the rivers and mariculture, contributed significantly to ARGs pollution in coastal areas (Zhang et al., 2018). ARGs conferring resistance to tetracyclines, sulfonamides, quinolones, macrolides, aminoglycosides and $\beta$-lactams were detected with high abundance of in water and sediments (Dang et al., 2017; Jia et al., 2018). It has been confirmed that ARGs were prevalent and abundant in both seawater and marine sediments in coastal areas of Bohai Bay, and coastal environment was likely the source of ARGs pollution in the ocean (Zhang et al., 2018). It is imperative to obtain more information on the distribution of ARGs in coastal areas and ocean. However, only a few research works have been published on the prevalence of ARGs in seas by real-time PCR (qPCR) quantification (Chen et al., 2019; Lu et al., 2019) and metagenomics (Yang et al., 2019a; Yang et al., 2019b).

As a typical semi-enclosed continental shelf sea and one of the most densely populated and industrialized zones in China (Song and Duan, 2019), the Bohai Sea areas have been affected greatly by the discharge with large amounts of pollutants, in which landbased pollutions accounted for $80 \%$ (Duan et al., 2010). Large discharge from $>40$ rivers and 99 land-based sewage outfalls flowed into the Bohai Sea (Song and Duan, 2019). Although antibiotic resistance was a natural phenomenon (D'Costa et al., 2011), the spread of ARGs has been influenced by intensive anthropogenic activities to some extent. The investigations on the distribution of ARGs in aquatic environment in the Bohai Sea indicated that ARGs were prevalent and abundant with the maximum abundance up to $6.23 \times 10^{3}$ copies/mL, and it has been confirmed that ARGs were inclined to be accumulated in sediments (Lu et al., 2019). Therefore, it is indispensable to investigate the abundances and distribution of ARGs in sediments in the Bohai Sea, to provide supporting data for further analysis and the initial information for the formulation of effective strategies to control the pollution of antibiotic resistance.

To the best of our knowledge, no comprehensive investigations have been reported regarding the spatial distribution of ARGs in surface sediments in the Bohai Sea. Therefore, a large-scale sampling for sediments was performed in the Bohai Sea and surrounding coastal areas. The abundances of target 29 ARG subtypes, involving in 6 classes of ARGs (sulfonamides, tetracyclines, macrolides, quinolones, aminoglycosides and $\beta$ lactams), were quantified by qPCR. Meanwhile, class 1 integronintegrase gene (intI1), hmt-DNA and 16S rRNA gene were evaluated. The influence of environmental factors on the distribution of ARGs was investigated, and the co-occurrence patterns between ARGs and bacterial communities were determined to reveal the potential host bacteria of ARGs. This research aimed at providing a comprehensive investigation into the prevalence and distribution of ARGs in the Bohai Sea, the typical semi-enclosed sea.

\section{Materials and methods}

\subsection{Sampling sites and samples collection}

The Bohai Sea is a typical semi-enclosed continental shelf sea in China, surrounded by the coastline of nearly $3800 \mathrm{~km}$. It extends for $350 \mathrm{~km}$ from west to east and $450 \mathrm{~km}$ from north to south with the area of $77,000 \mathrm{~km}^{2}$. There are several typical bays in the Bohai Sea: Bohai Bay (west, about 15,900 $\mathrm{km}^{2}$ ), Liaodong Bay (north, about $10,000 \mathrm{~km}^{2}$ ) and Laizhou Bay (south, about $6000 \mathrm{~km}^{2}$ ). Bohai Bay is surrounded by the Bohai Economic Ring, which is one of the most important economic centers in China. Liaodong Bay is located in the north of the Bohai Sea, receiving discharges from lots of rivers. Laizhou Bay is the largest semi-closed bay of the Bohai Sea and the important "Shandong Peninsula Blue Economic Zone", with more than ten rivers flowing into the bay. A large-scale sampling was performed in the Bohai Sea and the coastal zone along the coastline (Fig. 1). A total of 28 sediment samples were collected in the Bohai Sea, including sampling sites located in the Liaodong Bay (BS01 - BS07), Bohai Bay (BS08 - BS14), Laizhou Bay (BS15 - BS19), Bohai Strait (BS20 - BS22) and the Central Bohai Sea (BS23-BS28). Moreover, 22 samples were collected in intertidal zone in coastal areas (CZS01 - CZS11 \& CZW01 - CZW 11) and 3 samples were collected in rivers (CZS06R - CZS08R). The coastal sampling sites included estuaries, tourist areas, maricultural zone and ports, which considered as important functional areas in coastal zone.

Sediments in the Bohai Sea were collected during voyage by the research vessel "CHUANGXIN YI" from August 18 to August 25 in 2018, and sediments in the coastal zone were collected in summer (September 11 to September 19, 2018) and winter (November 4 to December $17,2017)$. Sampling sites located in coastal zone were about $2-3 \mathrm{~m}$ to the sea, and river sediment samples were collected in rivers which were $3-8 \mathrm{~km}$ to the estuaries. Surface sediments in the sea were collected using stainless steel box samplers, and sediments in coastal zone were collected by sterile disposable syringe in triplicate. Next, all the samples were stored at cooler containers and transported to laboratory for further analysis immediately.

\subsection{Physicochemical analysis}

The basic characteristics of sediment samples were measured regularly. The sediment water content was determined by $w=m_{l} / m_{s}$, in which $m_{l}$ represents the weight of liquid water, and $m_{s}$ represents the drying sediment weight. The grain sizes of lyophilized sediments were measured by the laser particle analyzer (Mastersizer 2000F, Marlvern, UK). The sediment samples were freeze-dried and sieved (200 mesh) for the measurement of total carbon (TC) and total nitrogen (TN) using the elemental analyzer (Vario MACRO cube, Elmentar, Germany). In addition, water samples, collected from aquatic environment surrounding the sediments sampling sites, were filtered through $0.45 \mu \mathrm{m}$ glass fiber membrane filters (Merck Millipore Ltd., Ireland) for the measurement of ammonia $\left(\mathrm{NH}_{4}^{+}-\mathrm{N}\right)$, nitrate $\left(\mathrm{NO}_{3}^{-}-\mathrm{N}\right)$, nitrite $\left(\mathrm{NO}_{2}^{-}-\mathrm{N}\right)$, reactive phosphate $\left(\mathrm{PO}_{4}^{3-}-\mathrm{P}\right)$ and reactive silicate $\left(\mathrm{SiO}_{3}^{2-}-\mathrm{Si}\right)$ using the continuous flow analyzer (Auto Analyzer III, Seal, Germany).

\subsection{DNA extraction and quantification of target genes}

The total DNA extraction for lyophilized sediment samples $(0.5 \mathrm{~g})$ were conducted by TIANamp Soil DNA Kit (TIANGEN, Beijing, China) according to the instructions. The concentration and purity of extracted 


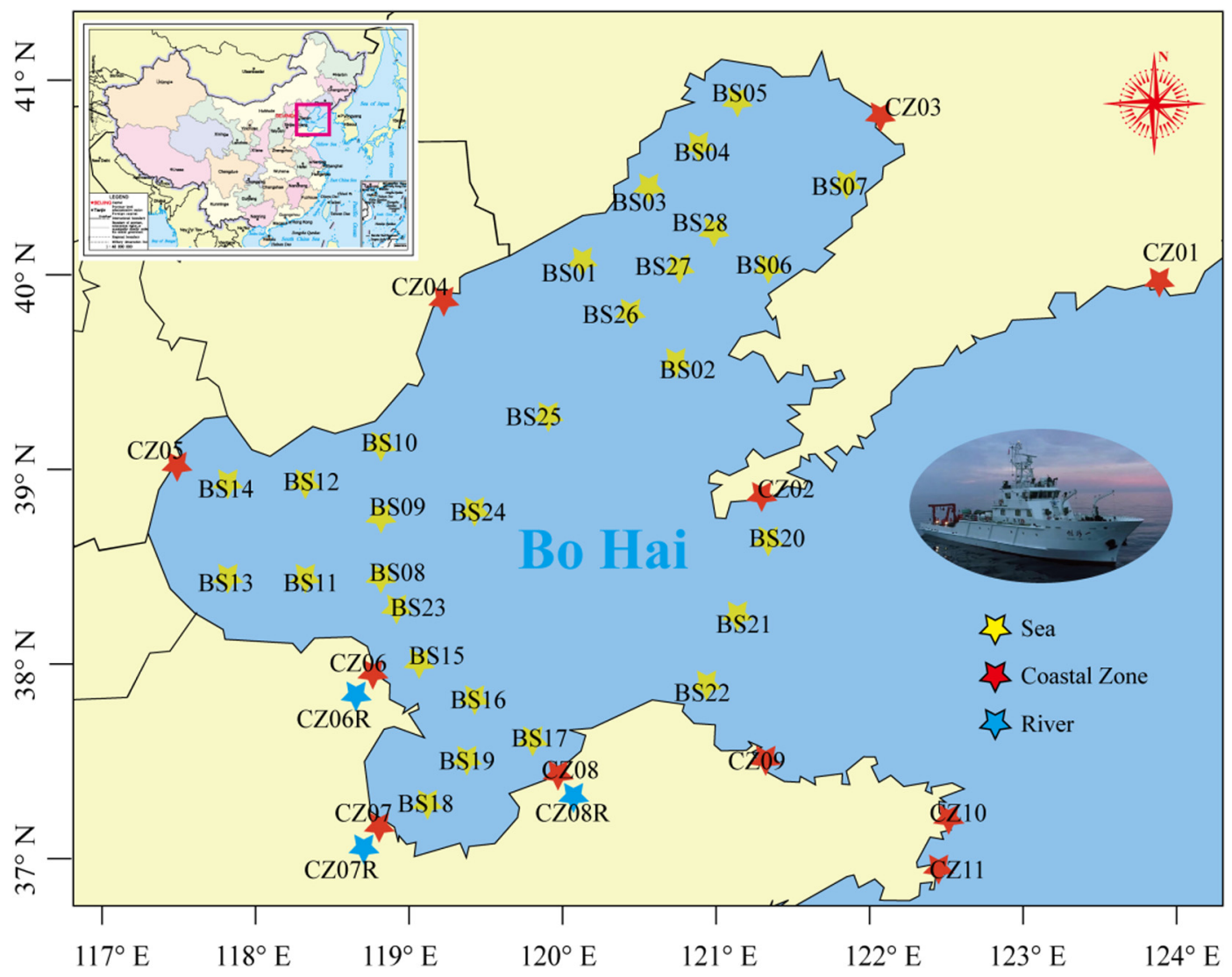

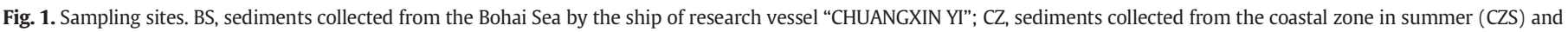
winter (CZW).

DNA were determined by NanoDrop Lite (Thermo Scientific, Wilmington, USA) and 1\% agarose gel electrophoresis.

Quantification of target ARGs, intI1, hmt and 16S rRNA gene in sediment samples were performed by real-time (qPCR) system (Bio-Rad CFX384 Touch, CA, USA). Six classes of ARGs were quantified in this research, including sulfonamides (sul1 and sul2), tetracyclines (tetB, tet $\mathrm{G}$ and tet $\mathrm{X}$ ), macrolides (erm $\mathrm{F}$ and erm $\mathrm{T}$ ), quinolones (qnrA, qnrB and qnrS), aminoglycosides ( $\operatorname{aac}\left(6^{\prime}\right) \mathrm{I} 1, \operatorname{aac}\left(6^{\prime}\right)-\mathrm{Ib}, \operatorname{aacC} 3$, aadA-01, aadA-02, aadA2-02, aadA2-03, aadA5, strA and strB), and $\beta$-lactams (blaOXA1, blaOXA2, blaOXA10, blaOXA30, blaTEM, blaGES, blaCMY, ampC and cphA) (Table S1). These ARGs involved in five different resistance mechanisms: antibiotic efflux, antibiotic inactivation, antibiotic target replacement, antibiotic target alteration and antibiotic target protection.

The qPCR reaction was performed with $10.0 \mu \mathrm{L}$ mixture $(5.0 \mu \mathrm{L}$ SYBR® Premix Ex Taq ${ }^{\mathrm{TM}}$ II, $0.4 \mu \mathrm{L}$ forward primer, $0.4 \mu \mathrm{L}$ reverse primer, $1.0 \mu \mathrm{L}$ DNA template and $3.2 \mu \mathrm{L} \mathrm{ddH}_{2} \mathrm{O}$ ) in triplicate. The qPCR amplified reactions were performed using Bio-Rad CFX384 system with the program as previous study (Lu et al., 2020a, 2020b): 30 s at $95^{\circ} \mathrm{C}$ (denaturation), 40 cycles for $5 \mathrm{~s}$ at $95{ }^{\circ} \mathrm{C}$ (denaturation), $30 \mathrm{~s}$ at annealing temperature and $40 \mathrm{~s}$ at $72{ }^{\circ} \mathrm{C}$ (extension). The melting curve was performed with temperature in the range of $60^{\circ} \mathrm{C}$ to $95^{\circ} \mathrm{C}$, and the melting curve was considered as specific amplification when it was detected with a single peak. All the target genes were amplified with two-steps method, while qnrA was amplified by three-steps protocols. Quantification of target genes were conducted based on the calibration standard curves of plasmids DNA with the abundance ranging from $10^{\circ}$ to $10^{7}$ copies $/ \mu \mathrm{L}$ (ten-fold dilution serial). The plasmids DNA standards were constructed by pMD19-T vector by the methods of TA cloning following instructions of pMD®19-T Vector (TaKaRa) (Lu et al., 2019). When the $\mathrm{R}^{2}$ value was higher than 0.99 and the amplification efficiencies ranged from $90 \%$ to $110 \%$, the amplification was considered to be effective. The unit of ARGs in lyophilized sediments was represented by "copies/g".
Additionally, the relative abundance of target genes was calculated by the equation:

\section{Relative abundance \\ $=$ Copy number of target gene/Copy number of 16S rRNA gene}

\subsection{High-throughput sequencing}

The purified DNA was sent to Majorbio (Shanghai, China) for highthroughput sequencing (Illumina, San Diego, USA) according to the standard procedures in Majorbio. The hypervariable regions (V4-V5) of bacterial 16S rRNA genes were amplified by PCR system (GeneAmp 9700, ABI, USA) with the forward primer 515F (5'-GTGCCAGCMGCCG CGG-3') and the reverse primer 907R (5'-CCGTCAATTCMTTTRAGTTT$\left.3^{\prime}\right)$. Raw data of PE300 pair-end sequencing were merged, qualityfiltered and OTU (operational taxonomic units) clustered using Flash (version 1.2.11), Qiime (version 1.9.1) and Uparse (version 7.1). The 16S rRNA gene sequences were classified and annotated by RDP classifier (version 2.11) according to the Silva database, and the statistics of OTUs was carried out by Usearch (version 7.0). Additionally, raw reads were deposited into the NCBI (National Center for Biotechnology Information) database (Accession Number: SRP233433).

\subsection{Statistical analysis}

Calculations and plotting were performed using Microsoft Office Excel 2016 and Origin 2017 (Origin Lab Corporation, USA). Correlation analysis was conducted by SPSS Statistics (version 19, IBM, USA). Contour map was accomplished by Surfer (version 11, Golden Software, USA). Principal component analysis (PCA) was accomplished by Paleontological Statistics (PAST, version 3.25) and redundancy analysis (RDA) 
was conducted by Canoco 5 (Šmilauer and Lepš, 2014). Circos analysis was performed by Circos-0.67-7 and network analysis was visualized by Cytoscape 3.7.1.

\section{Results and discussion}

\subsection{Abundances of $16 S$ rRNA gene, intI1 and total ARGs}

In the typical semi-enclosed continental shelf sea, the marine environment might be poorly flushed, the space was relatively closed and the seawater movement was relatively weaker compared to open seas. Furthermore, the Bohai Sea area was one of the most highly industrialized and urbanized zones in China. Because of the unique characteristics and the densely populated geographical location, the Bohai Sea area has been affected by large amounts of pollutants. Therefore, it could be speculated that the contaminant might be trapped in the sea (Song and Duan, 2019). According to previous investigations, the abundance of ARGs in the Bohai Sea was higher than that in the East Sea (Chen et al., 2019) and the Yellow Sea (Lu et al., 2019) in China. Therefore, the Bohai Sea was set as the objective in this research to investigate the spatial distribution of 16S rRNA gene, intI1 and ARGs. The study areas in the sea included the Liaodong Bay, the Bohai Bay, the Laizhou Bay, the Bohai Strait and the central sea basin, and the coastal study areas involved in the typical estuaries (CZ01, CZ03, CZ05, CZ06, CZ07 and CZ08), tourist areas (CZ02, CZ04 and CZ09), maricultural areas (CZ10 and CZ11) and ports (CZ05). These functional zones have been considered as the potential reservoirs of ARGs in coastal areas (Lu et al., 2019).

The 16S rRNA gene was well studied and used as biomarkers for normalizing quantitative data for bacteria (Pei et al., 2006). As shown in
Fig. 2, the absolute abundance of 16S rRNA gene was in the range of $5.45 \times 10^{6}$ copies/g (BS08) to $1.67 \times 10^{8}$ copies/g (BS17) in the sea, and it varied from $5.66 \times 10^{6}$ copies $/ g$ (CZO1) to $3.01 \times 10^{8}$ copies $/ \mathrm{g}$ (CZ11) in coastal zone. The abundance of 16S rRNA gene quantified the bacterial cells, indicating the varying bacterial abundance at different sites, especially in the coastal zone. It was worthy to note that the bacterial 16S rRNA gene was more abundant in BS15-BS19 than that in other areas, which further demonstrated that the microbial concentration was significantly higher in the Laizhou Bay $(\mathrm{p}<0.05)$.

Integrons played important roles in the horizontal transfer of ARGs by bacteria (Yuan et al., 2019). The levels of intI 1 from the sediments was determined to reveal the transfer ability of ARGs. The result showed that intI1 was detected with higher abundance $\left(2.83 \times 10^{5}\right.$ to $2.51 \times 10^{8}$ copies/g) in coastal areas, while that detected in the sea was relatively lower $\left(9.96 \times 10^{3}\right.$ to $5.79 \times 10^{7}$ copies $\left./ g\right)$. For further analysis, the intI1 abundances were higher than $10^{6}$ copies/g in B11-B13 in the Bohai Bay. Meanwhile, it was detected with relatively higher abundance in B17-B19 in the Laizhou Bay $\left(5.43 \times 10^{5}\right.$ to $7.85 \times 10^{5}$ copies/g). Instead, the abundance of intI1 in the Liaodong Bay varied greatly, which were generally lower than $4.35 \times 10^{5}$ copies/g. In general, the levels of intI1 demonstrated the tendency of the Bohai Bay > Laizhou Bay $>$ Liaodong Bay $(\mathrm{p}<0.05)$. Additionally, it remained stable in the Bohai Strait and the central sea basin with relatively lower abundance ( $5.05 \times 10^{4}$ to $1.64 \times 10^{5}$ copies $/ g$ ). Interestingly, the abundance of intI 1 ascended one order of magnitude in rivers than that detected in corresponding estuaries and surrounding sea areas, which was consistent with the ARGs distribution in aquatic environment in the Bohai Sea areas (Lu et al., 2019). For example, intI1 was detected with the tendency of CZS07R $\left(2.29 \times 10^{7}\right.$ copies $\left./ g\right)>$ CZS07 $\left(1.53 \times 10^{6}\right.$ copies/ g) $>\operatorname{BS} 18\left(5.43 \times 10^{5}\right.$ copies $\left./ g\right)$ and CZS08R $\left(2.51 \times 10^{7}\right.$ copies/

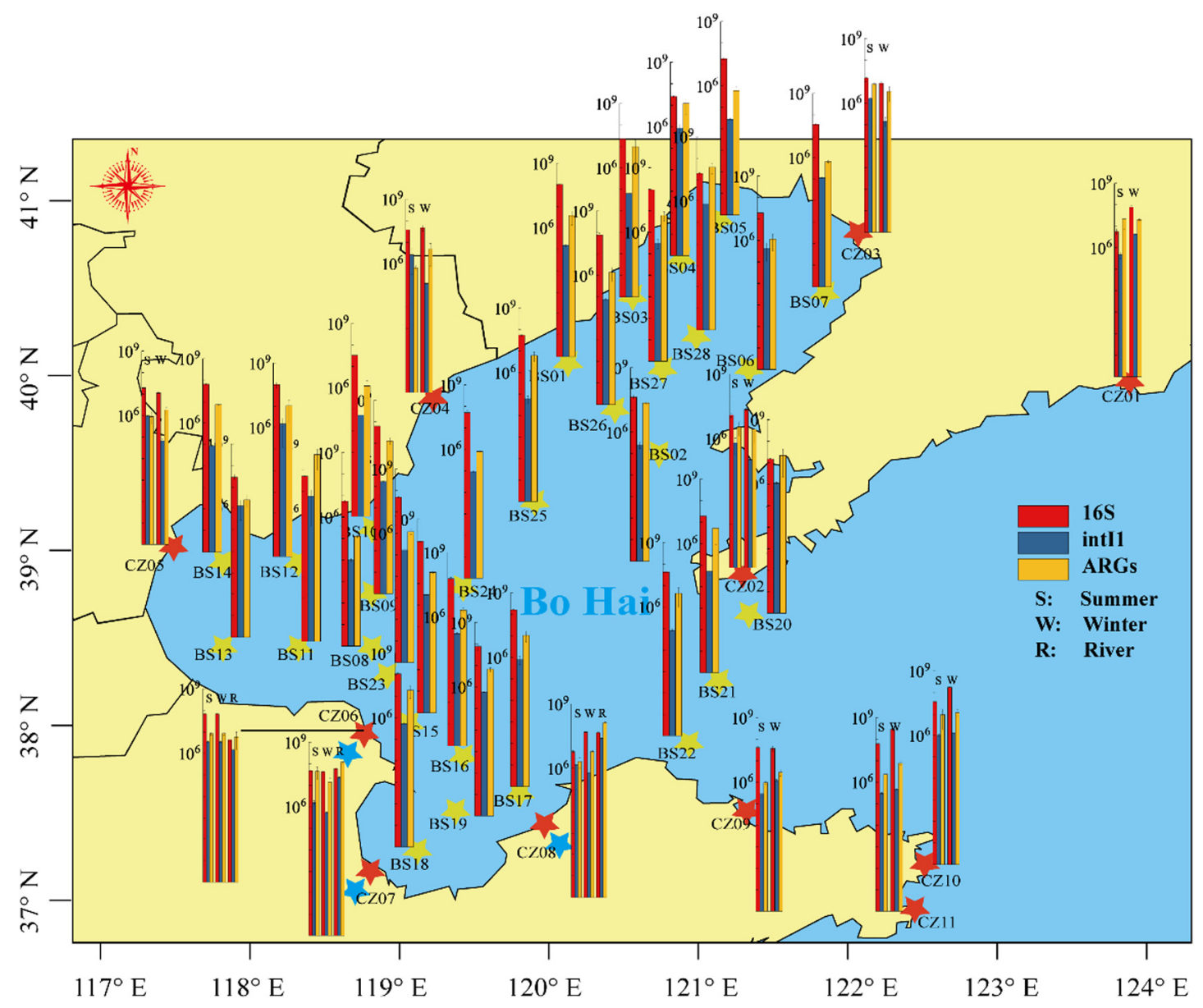

Fig. 2. Absolute abundance of $16 \mathrm{~S}$ rRNA gene, intI1 and the total absolute abundance of target antibiotic resistance genes (ARGs). 
g) $>$ CZS08 $\left(1.50 \times 10^{6}\right.$ copies/g $)>$ BS17 $\left(7.85 \times 10^{5}\right.$ copies/g $)$. There existed a distinct gradient from the rivers to the estuaries, and to the sea, indicating the influence of land-based pollution on the sea, as the intI1 gene was marker of selective pressures imposed by anthropogenic pollution (Gillings et al., 2015).

The abundance and spatial distribution of total target 29 ARG subtypes in the Bohai Sea areas was exhibited in Fig. 2. These target ARGs were detected with higher detection frequencies and abundances in marine environment (Chen et al., 2019). In the sea, the total ARGs abundance varied over 3 orders of magnitude between different sites, which was at the maximum in BS11 $\left(4.94 \times 10^{8}\right.$ copies $\left./ g\right)$ and the minimum in BS08 $\left(1.27 \times 10^{5}\right.$ copies $\left./ g\right)$. Among most of the sediment samples, the ARGs were detected with the absolute abundance in the range of $10^{6}$ to $10^{7}$ copies/g. In coastal areas, the total absolute abundance of target ARGs topped at CZSO7 (4.44 $\times 10^{7}$ copies/g), which was the estuary of Xiaoqing River. In contrast, the ARGs in tourist areas was detected with relatively lower absolute abundance, such as CZS04, CZS05 and CZS09. More interestingly, ARGs detected in the Xiaoqing River and Jiehe River were more abundant than those in their estuaries (CZS07R $>$ CZS07 and CZS08R $>$ CZS08), while the ARGs abundance in the Yellow River estuary was higher than that in the Yellow River (CZS06R $<$ CZS06). Riverine ecosystems were the receiving environments of the discharge from anthropogenic activities, which were the important sources of ARG contamination. As reported, the richness and abundance of ARGs were significant high in the downstream area of Houxi River as a result of the high population density in Jimei district (Peng et al., 2019). Correspondingly, the higher abundance of ARGs in the Yellow River estuary might be related to the intensive humanity activities in the Yellow River Delta. To the contrary, the relatively lower ARGs abundance in the estuaries of Xiaoqing River and Jiehe River might be attributed to the highly dynamic nature and the dilution of seawater in estuarine ecosystems.

\subsection{Spatial distribution of different classes of ARGs}

As the wide use of antibiotics of sulfonamides, tetracyclines, macrolides, quinolones, aminoglycosides and $\beta$-lactams, the corresponding ARGs have been detected in various environments. The spatial distribution of these classes of ARGs in the semi-enclosed sea was demonstrated in Fig. 3. The absolute abundance averages of sulfonamides and quinolones resistance genes were at the level of $10^{6}$ copies $/ \mathrm{g}$, while abundances of $q n r$ fluctuated greatly in different samples. Tetracyclines resistance genes were detected with the absolute abundances at the average of $10^{5} \mathrm{copies} / \mathrm{g}$, and the abundance averages of macrolides, aminoglycosides and $\beta$-lactams resistance genes kept at the level of $10^{4}$ copies/g.

The class of sulfonamides resistance genes was the most prevalent and abundant class of ARGs, with the absolute abundance ranging from $5.65 \times 10^{4}$ copies/g (BS08) to $1.84 \times 10^{7}$ copies/g (BS18) in the sea and coastal zone. As shown in the contour map, the sul genes were more abundant in the Laizhou Bay and the mouth of Bohai Bay. Similarly, tetracyclines resistance genes also hold high absolute abundances in the range of $6.47 \times 10^{4}$ copies/g (BS08) to $2.09 \times 10^{7}$ copies $/ g$ (BS02), which exhibited higher abundances in the Bohai Bay, the mouth of Liaodong Bay and the central sea basin. Quinolones resistance genes varied considerably from $1.98 \times 10^{2}$ copies/g in BS10 to $4.92 \times 10^{8}$ copies/g in BS11, with relatively higher abundance in the Laizhou Bay and parts of the Bohai Bay and Liaodong Bay. Moreover, macrolides, aminoglycosides and $\beta$-lactams resistance genes, detected with greatly varied absolute abundances, demonstrated relatively higher abundances in the Laizhou Bay and the south of Bohai Bay. The higher abundance of macrolides, aminoglycosides and $\beta$-lactams resistance genes in the eastern of central sea basin was worthy to be noted. Taken into all the target ARGs, it exhibited relatively higher levels of ARGs pollutions in the Laizhou Bay, the Bohai Strait, and parts of the Bohai Bay and the Liaodong Bay, which have been confirmed as the regions influenced seriously by human activities. The variation in the prevalence of ARGs might be caused by the land-based and marine-based pollutions from complex anthropogenic impacts. Laizhou Bay was one of the important maricultural zones in China, receiving amounts of nutrient substances and contaminants from the Yellow River. Bohai Bay, receiving large discharge from the Haihe River and the Yellow River, was an important breeding base of sea cucumbers with the abuse of antibiotics as the growth promoters. In Liaodong Bay, the marine culture for scallop, abalone, prawn, and sea cucumber has been well known for a long time. Besides, these areas received the discharge from industrial bases, pharmaceutical bases, wastewater treatment plants, etc. Moreover, the Bohai Strait was an indispensable shipping channel in the Bohai Sea influenced by intensive anthropogenic activities (Song and Duan, 2019). Therefore, the interaction of land-based pollutions and marine-based pollutions, and the intensive anthropogenic activities contributed to the different spatial distribution of ARGs.

Unlike the spatial distribution of ARGs in the water samples, which demonstrated that the ARGs abundances detected in coastal water samples were higher than those in the water samples from the Bohai Sea (Lu et al., 2019), there were no significant differences in ARGs abundances of sediments between the marine and the coastal areas ( $p>0.05)$. The different trends of ARGs in sediment and water samples were probably due to the contrast between the steady state of sediments and the complex hydrodynamic conditions of water phase. Complicated environmental factors (e.g., intensive anthropogenic activities, interaction of land-based and marine-based pollutions) contributed to the differences. It has been confirmed that ARGs were inclined to be accumulated in sediments (Lu et al., 2019), so that the influence of seawater variation on the sediments was not significant, further indicating that ARGs in the sediments were abundant and enduring. Sediments were important ARGs reservoirs, and the abnormal existence of ARGs could be used as the indicators for anthropogenic impacts (Chen et al., 2019). Previous researches demonstrated that the scenario in coastal environment (e.g., Pearl River Estuary) was complex due to the various potential sources of ARGs (Chen et al., 2013a; Lu et al., 2019). The pollution status caused by human activities in the sea was relatively weaker than that in coastal areas. The abundances and diversity of ARGs detected in the sediments collected from the deep ocean have been confirmed to be lower than that in coastal sediments (Chen et al., 2013b). Therefore, the equal level of ARGs contamination in sediments from the sea and coastal areas revealed that the surface sediments in the sea had been contaminated by ARGs to some extent.

\subsection{Absolute and relative abundances of ARG subtypes}

A total 29 ARG subtypes were quantified and demonstrated in the heat maps (Fig. 4). Most of the target ARG subtypes were detected with the detection frequencies of $100 \%$, except that the ARG subtypes of qnrA, qnrS, blaOXA1, blaOXA2, blaOXA30 and blaGES were detected with the frequencies of $86 \%, 93 \%, 93 \%, 93 \%, 86 \%$ and $72 \%$. In general, the ARG subtypes of sulfonamides were detected with the highest abundances, followed by the ARG subtypes of tetracyclines. The ARG subtypes of macrolides, quinolones and aminoglycosides differed significantly in different sediment samples with relatively lower absolute abundance, and $\beta$-lactams resistance genes hold the lowest abundances with great variation.

For the ARG subtypes encoding resistance to sulfonamides, both sul1 and sul2 hold high abundances in the range of $10^{4}-10^{7}$ copies/g and topped at BS18, which was located near the shore in the Laizhou Bay. Such decrease of sul genes from near-shore to off-shore areas has been determined in the East China Sea Bay (Chen et al., 2019). Gene tetX was detected with higher abundances $\left(3.26 \times 10^{4}-2.09 \times 10^{7}\right.$ copies/g) among tetracyclines resistance genes, in which several samples with the abundances nearly $10^{7}$ copies/g were collected from the Liaodong Bay. The tet $\mathrm{G}$ and tetB genes were detected with lower abundances. The different abundances of tet genes probably resulted from 
A. Sulfonamide

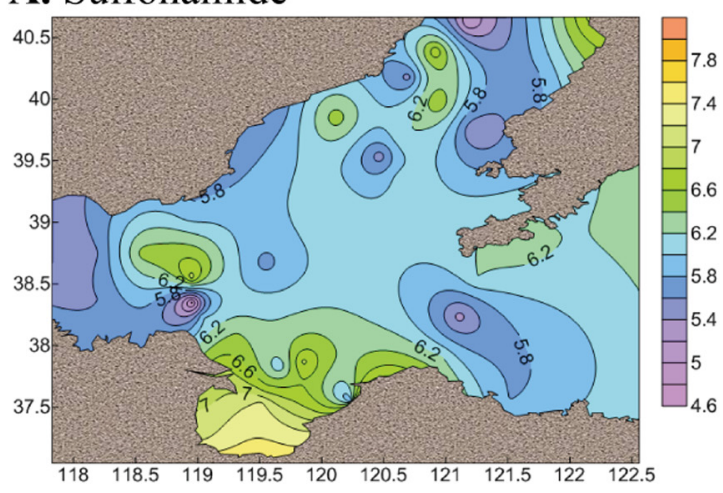

C. Macrolide

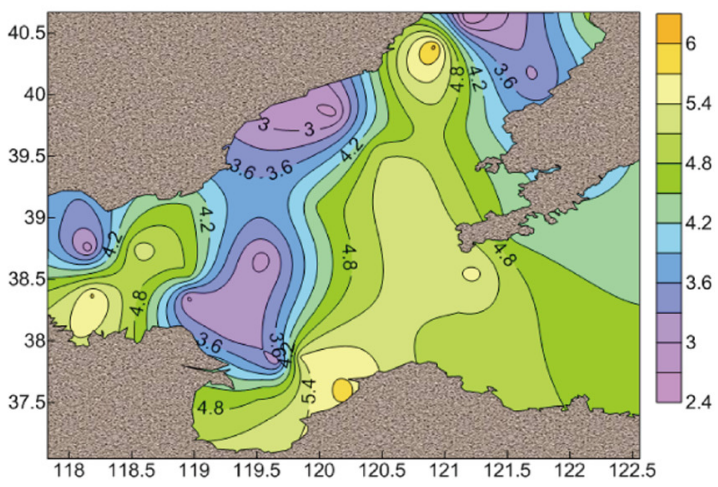

E. Aminoglycoside

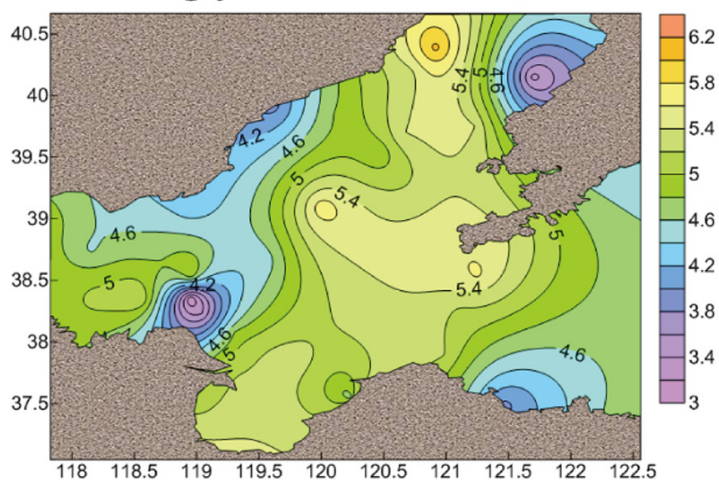

B. Tetracycline

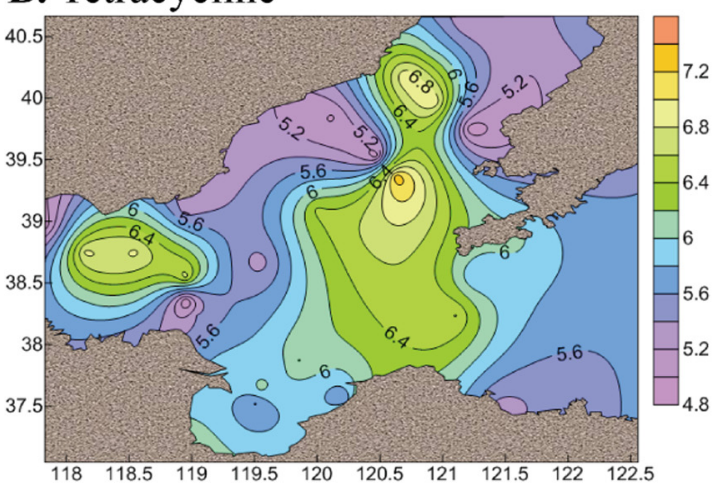

D. Quinolone

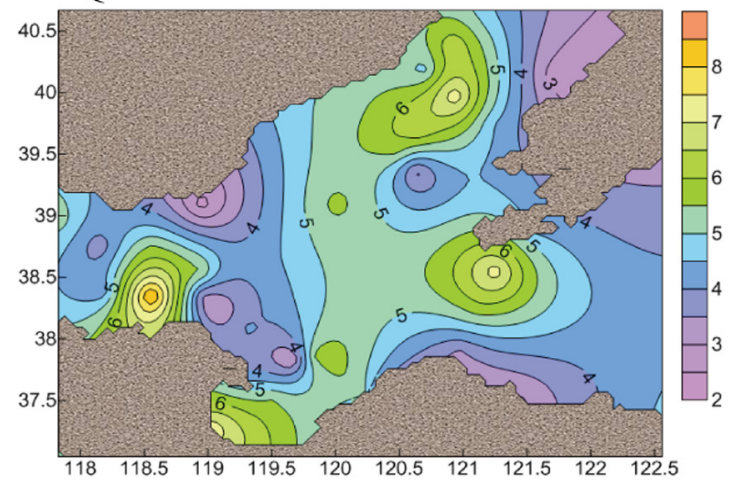

F. $\beta$-Lactamase

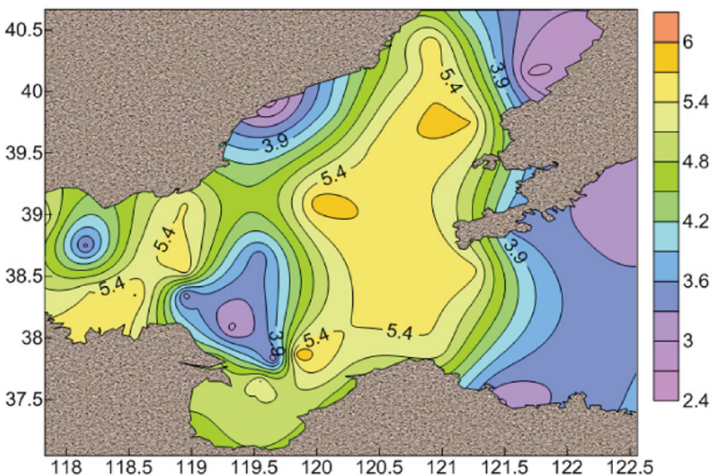

Fig. 3. Spatial distribution of different classes of antibiotic resistance genes (ARGs).

their different antibiotic resistance mechanisms. The resistance mechanism of tetX was antibiotic inactivation while the resistance mechanism of tetB and tet $\mathrm{G}$ was antibiotic efflux (Lu et al., 2019). For microbes, the direct inactivation could be considered as one of the most effective ways to react to the antibiotics in highly impacted environments by ARGs (Chen et al., 2013b). The absolute abundances of ermF and ermT, encoding resistance to macrolides, topped at BSO2 and BS04 in the Liaodong Bay, respectively. Quinolones resistance genes were detected with great variations, especially the $q n r A$ gene (up to $4.91 \times 10^{8}$ copies/g in BS11), while the genes of $q n r B$ and $q n r S$ both topped at BS04. Several samples with relatively higher abundances of qnr genes were collected from the north of Bohai Strait, the Central Bohai Sea and the mouth of Liaodong Bay. The absolute abundances of ARG subtypes encoding resistance to aminoglycosides and $\beta$-lactams fluctuated greatly in different samples. In terms of aminoglycosides resistance genes, strB was detected with the highest abundances, followed by aadA and strA. As for $\beta$-lactams resistance genes, ampC (up to $4.61 \times 10^{5}$ copies/g) and
blaOXA10 (up to $3.82 \times 10^{5}$ copies/g) were more abundant than other ARG subtypes. Similarly, the absolute abundances of ARG subtypes encoding resistance to macrolides and $\beta$-lactams detected in the East Sea were at lower levels than those encoding resistance to sulfonamides and tetracyclines (Chen et al., 2019).

The spatial variability of different ARG subtypes might be attributed to the different sources of ARGs from human activities on land and in the sea. Sulfonamide and tetracycline antibiotics have been widely used in aquacultural systems as growth promoters. Several wellknown maricultural bases were located in the Bohai Sea. Besides, the persistence of ARGs was longer than the antibiotics themselves (Chen et al., 2019). Therefore, the ARG subtypes of sul and tet were predominant in the Bohai Sea areas. The subtypes of sul genes have been confirmed to be the most prevalent and abundant ARG subtypes due to their high persistence in environments. The result was consistent with the investigations on the Bohai Bay (Zhang et al., 2018), the East Sea (Chen et al., 2019) and Pearl River Estuary (Chen et al., 2013a). 


\section{A}

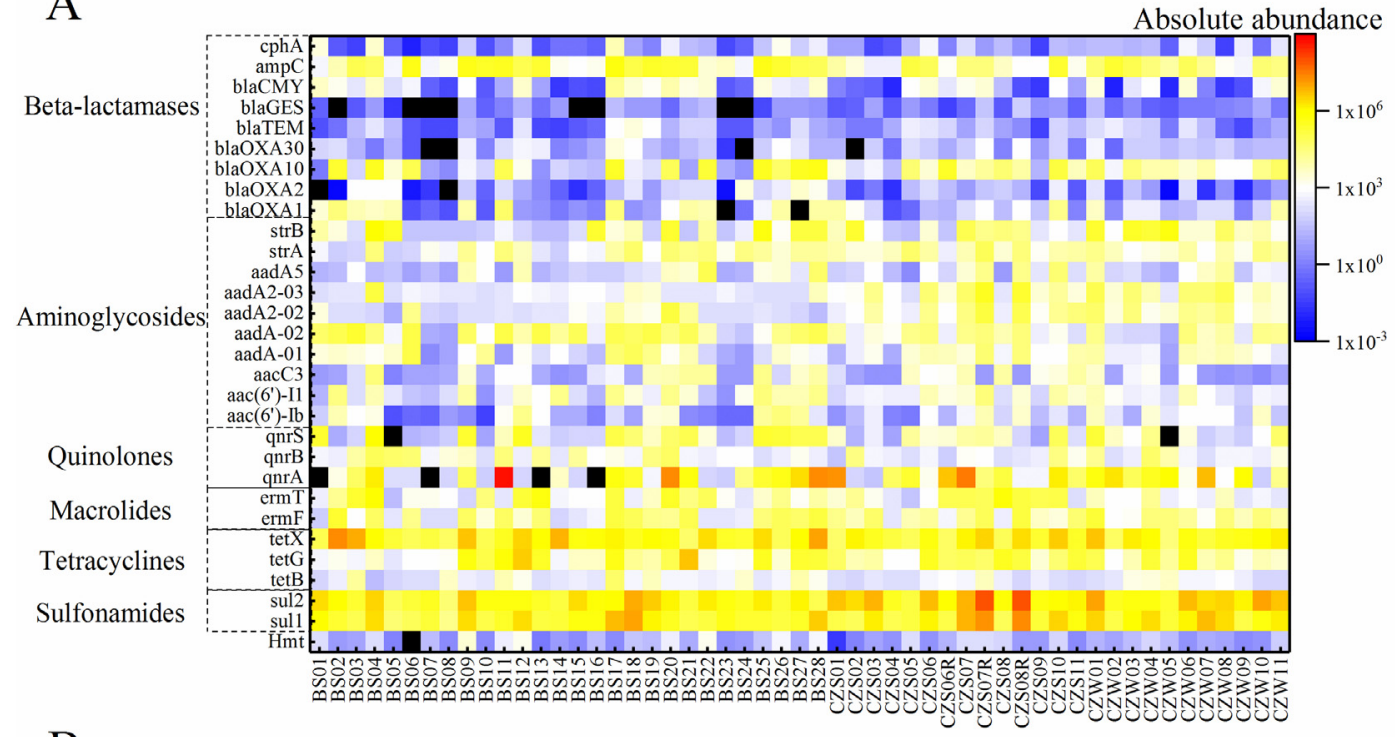

B

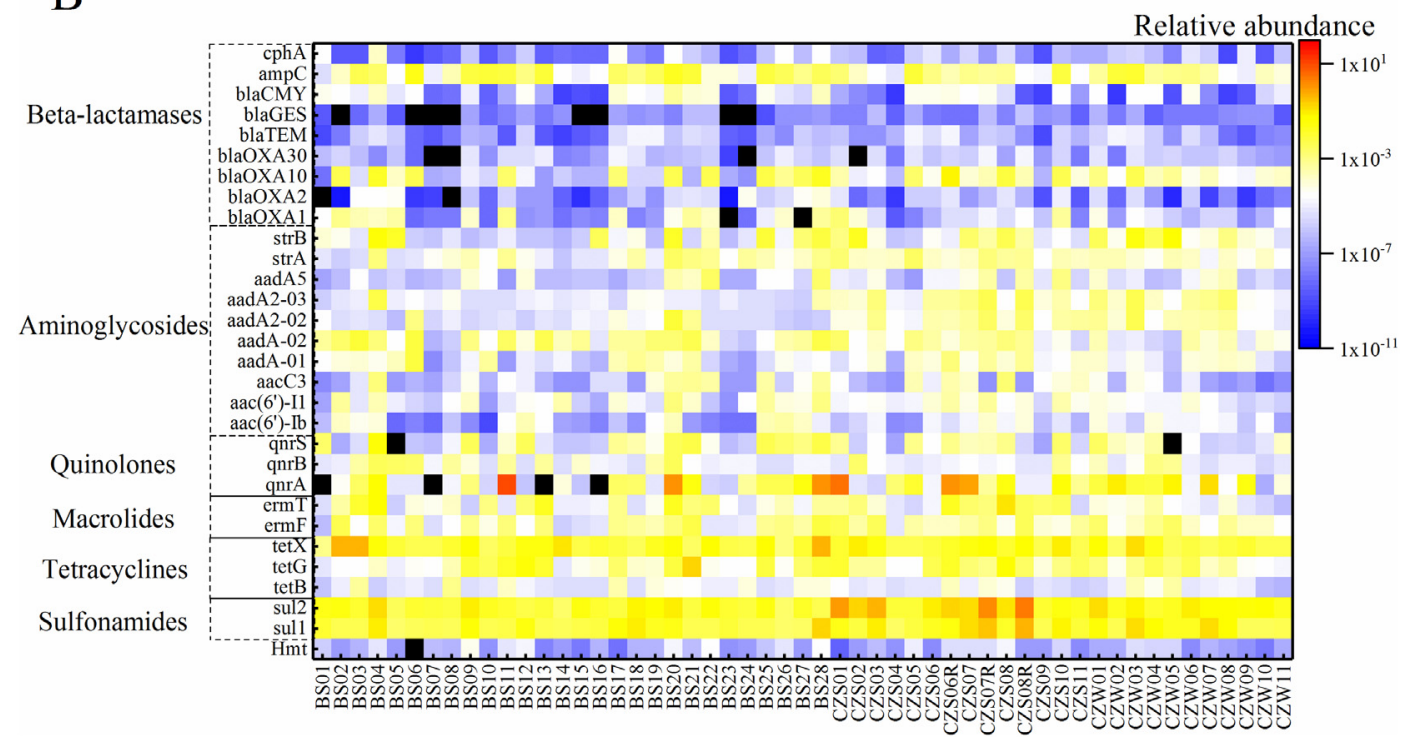

Fig. 4. Absolute abundance of ARGs (A) and relative abundance (ARGs copies/16S rRNA gene copies) of ARGs (B).

Furthermore, the antibiotics of quinolones, aminoglycosides and $\beta$ lactams have often been used in clinic, resulting in the enrichments of ARGs resistant to antibiotics of aminoglycosides, $\beta$-lactams, quinolones, sulfonamides, tetracyclines and macrolides (Chen et al., 2018). As a result, the discharge from maricultural systems, industrial districts, pharmaceutical bases and the wastewater treatment plants might be the sources of ARGs.

The relative abundances of target ARG subtypes were calculated and exhibited in Fig. 4B, in order to eliminate the influence of biomass. Sulfonamides resistance genes were quantified with the relative abundances higher than $10^{-3}$ and the maximum of sul1 at $1.99 \times 10^{-1}$. In the East China Sea Bay, the sul1 gene showed relatively lower abundance with the highest at $1.29 \times 10^{-2}$ (Chen et al., 2019). Among tetracycline resistance genes, the relative abundances of tet $\mathrm{X}$ were generally higher than $10^{-3}$, while tet $\mathrm{B}$ and tet $\mathrm{G}$ were detected with lower relative abundances in the range of $10^{-6}-10^{-3}$ and $10^{-5}-10^{-1}$, respectively. Moreover, the relative abundances of ARG subtypes encoding resistance to quinolones and macrolides varied from $10^{-6}$ to $10^{-2}$ in general. The relative abundances of aminoglycosides resistance genes significantly varied from $10^{-7}$ to $10^{-3}$. Furthermore, $\beta$-lactams resistance genes were detected with the lowest relative abundances, which were roughly ranged from $10^{-10}$ to $10^{-4}$. The variations of the relative abundances of ARG subtypes in different sediment samples was generally consistent with those of the absolute abundances, with only a few differences were identified. As shown in Fig. S1, there were strong and significant correlations between the absolute abundances and relative abundances $(p<0.01$ ). The relative abundances of ARG subtypes detected in CZS01 was secondary to that in BS11, while the absolute abundances of ARG subtypes in CZS07 was second to BS11. It was worth noting that the relative and absolute abundances were both high in the samples of BS11, BS20, BS28, CZS01 and CZS07, further confirming that the quantitative methods of absolute quantification and relative quantification were both feasible and receivable for further researches. It was reasonable to investigate the ARGs distribution based on the absolute abundances or relative abundances of ARGs.

As the coastal zone was more susceptible to land-based discharge and pollution (Lu et al., 2019), the sediment samples in coastal areas were collected in summer and winter. The abundances of target ARGs showed different seasonal trends in different sampling sites. A total of 11 sediment samples and 29 target ARG subtypes were taken into account. It showed that $57.9 \%$ of the target ARG subtypes were more abundant in winter, while $42.1 \%$ of them were more abundant in summer. 
The result demonstrated that spatial differences overwhelmed seasonal differences in ARGs abundance, which has been also determined in a riverine ecosystem in Xiamen city (Peng et al., 2019). It has been confirmed that different estuaries demonstrated different seasonal trends in the ARGs abundance due to the fickle seasonal nature of pollutant discharge from the rivers and the various behaviors of estuarine sediment ARGs (Lu and Lu, 2020). Besides, previous research suggested that the temperature didn't have significant effects on the distribution of ARGs in coastal areas and it demonstrated that ARGs in coastal areas along the Bohai Sea were relatively abundant in winter (Lu et al., 2020a, 2020b). The variation of ARGs as a result of environmental fluctuations was probably induced by multiple human activities to some extent.

The investigations on ARGs in the Bohai Bay demonstrated that the contribution of river runoff to the distribution of ARGs in coastal areas might be higher than that of mariculture (Zhang et al., 2018). ARGs in the sediments collected from the typical rivers and estuaries (Yellow River, Xiaoqing River and Jiehe River) demonstrated that most of the target ARGs were detected with higher abundances in the rivers than that in downstream estuaries, especially in the Xiaoqing River and Jiehe River. In addition, hmt-DNA, as a human mitochondrial marker indexing human activities on the distribution of ARGs, was quantified in this research. The absolute abundances of $h m t$ generally varied from 1.0 copies $/ g$ to $2.91 \times 10^{3}$ copies $/ g$, and the relative abundances differed from $4.62 \times 10^{-9}$ copies/g to $4.57 \times 10^{-5}$. The abundances of $h m t$ detected in the Bohai Sea was slightly lower than that in the Haihe River and the Tianjin Water Park, with the absolute abundances at $10^{5}$ to $10^{6}$ copies/g sediment and the relative abundances at $10^{-8}-10^{-2}$ copies/16S rRNA gene copies (Tan et al., 2018). Therefore, anthropogenic influences were important factors on the distribution of ARGs, and the discharge from rivers was one of the important sources of ARGs for receiving sea.

\subsection{Ordination analysis for different sediment samples}

The physicochemical properties of sediments and water have been confirmed to be related to the distribution of ARGs, including nitrogen, phosphorus, organic carbon and so on (Lu et al., 2019). Therefore, the ordination analysis was carried out to describe the influence factors on the distribution of ARGs. PCA analysis was performed and demonstrated in Fig. 5A \& B. The variance of component 1 (PC1) accounted for $60.4 \%$ with the significant distinction of BS11 for the high abundance of qnrA. The variance of PC2 accounted for 33.9\%, which explained the differences in most of the sediment samples, such as CZW10, CZW11 and BS17. Besides, the differences between the river sediment samples (CZS07R and CZS08R) and other sediment samples from the sea and coastal areas attributed to the variance of PC3 (5.5\%). The clustering result of PCA analysis further confirmed that there were no significant differences in the ARGs distribution, except for several typical samples.

It has been confirmed that environmental factors have significant effects on the distribution of ARGs. RDA analysis between ARGs and environmental factors was conducted based on the absolute abundances of ARGs. Many studies have been focused on the influence of organic pollutants and nutrient substances on the distribution of ARGs (Lu et al., 2020a, 2020b). The basic qualities of sediments (water content, grain size, TC and TN) and water (ammonia, nitrate, nitrite, phosphate and silicate) in corresponding aquatic environment were both measured and analyzed (Fig. 5C \& D). It was worthy to note that the sediment qualities played more important roles on the distribution of ARGs, while the contribution of water qualities was weaker. The relatively stable status of sediments and the strong convective motion of water resulted in the phenomena. The RDA model indicated that the water content significantly attributed to the distribution and variation of ARGs $(p<0.05)$. Grain size accounted for higher explanatory variables in coastal
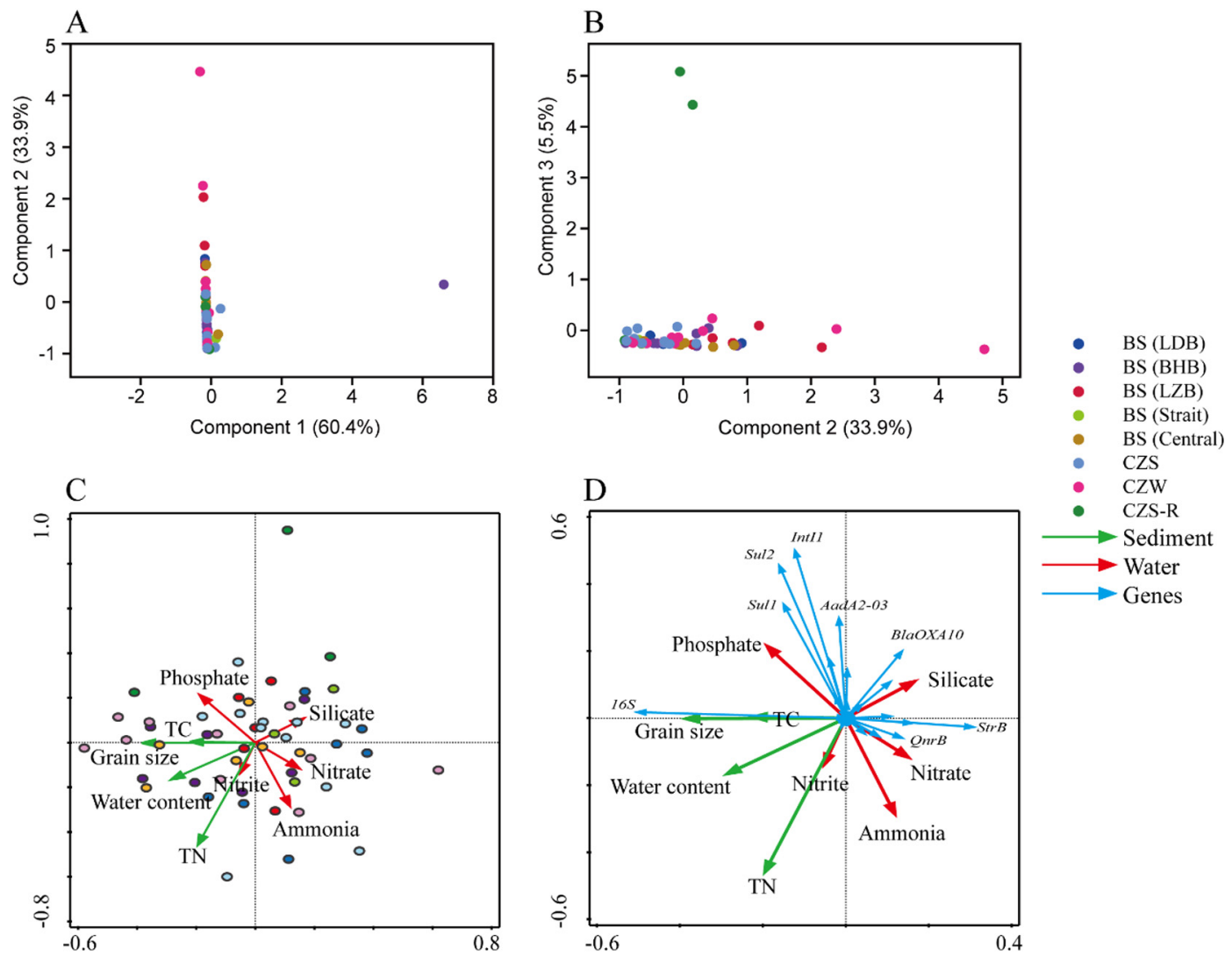

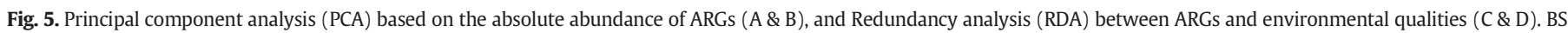
sediments in the Bohai Sea; CZS, sediments in coastal areas in summer; CZW, sediments in coastal areas in winter; CZS-R, sediments in rivers. 
sediments. Besides, the concentration of TN, phosphate and ammonia influenced the distribution of ARGs partly. Interestingly, 16S rRNA gene was closely correlated with the grain size of sediments, and sul1, sul2 and intI1 were positively correlated with the phosphate of water. This study revealed that the characteristics of sediments and seawater in corresponding aquatic environment have combined effects on the ARGs in sediments. Meanwhile, it is effective to reduce the discharge of conventional pollutants for controlling the ARGs risks.

\subsection{High-throughput sequencing for bacterial communities}

Illumina high-throughput sequencing was conducted to reveal the microbial communities (Fig. 6). A total of 1,344,617 optimized sequences were obtained from the selected 26 sediment samples. After subsampling to an equal depth and clustering with $97 \%$ similarity, 7060 operational taxonomic units (OTUs) were generated. The community richness estimators (Chao, Sobs and ACE) and the community diversity estimators (Simpson and Shannon) were showed in Fig. S2. The result indicated that the community richness and diversity were generally higher in the samples collected from the sea, while they fluctuated greatly in coastal sediment samples. Moreover, the high coverage $(>0.98)$ revealed that the sequencing depth was enough to represent the bacterial communities in sediments.

A total of 56 phyla of bacteria were detected in the sediments (Fig. 6A). Proteobacteria, Bacteroidetes, Firmicutes, Actinobacteria, Planctomycetes, Acidobacteria and Chloroflexi were the dominant phyla. Most of the sediment samples were dominated by the phyla of Proteobacteria and Bacteroidetes. Several samples were dominated by Firmicutes, for example, BS06 of $60.9 \%$, BS07 of $41.6 \%$, BS17 of $43.8 \%$, BS20 of $30.0 \%$, CZS07 of 41.3\% and CZSO7R of 48.3\%. Besides, Actinobacteria were detected with higher relative abundances in CZS01 (25.3\%) and CZSO8R (34.5\%), while Planctomycetes were detected with higher relative abundance in BS03 (13.7\%). On genus level, a total of 1143 genera were detected and the dominant genera were exhibited in Fig. 6B. In total, the genera of Gillisia,
A
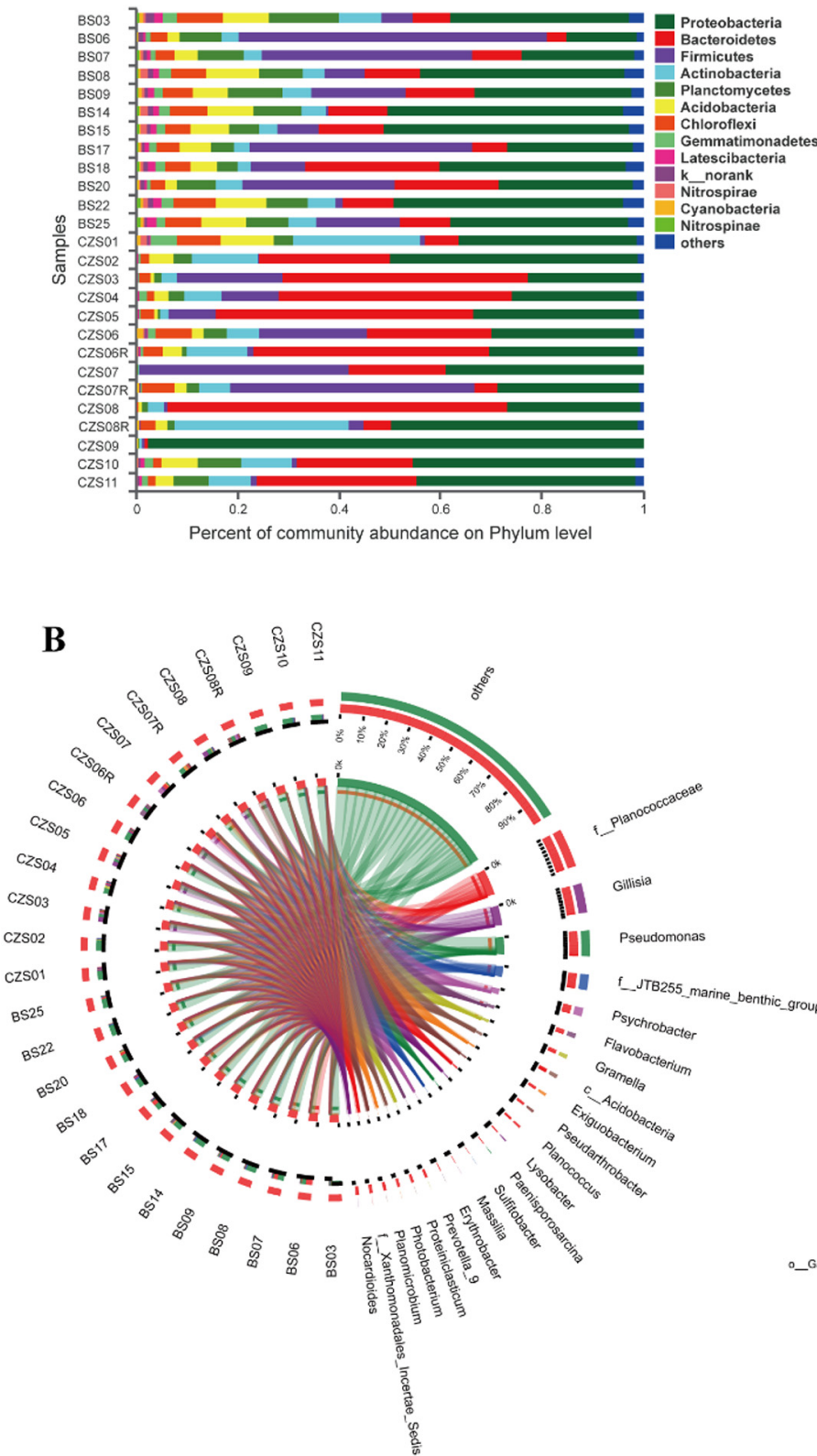

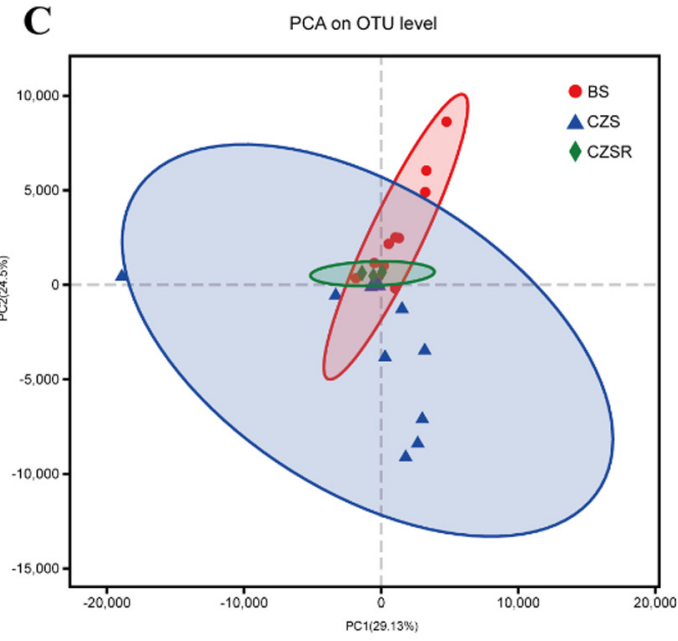

D

Kruskal-Wallis $\mathrm{H}$ test bar plot

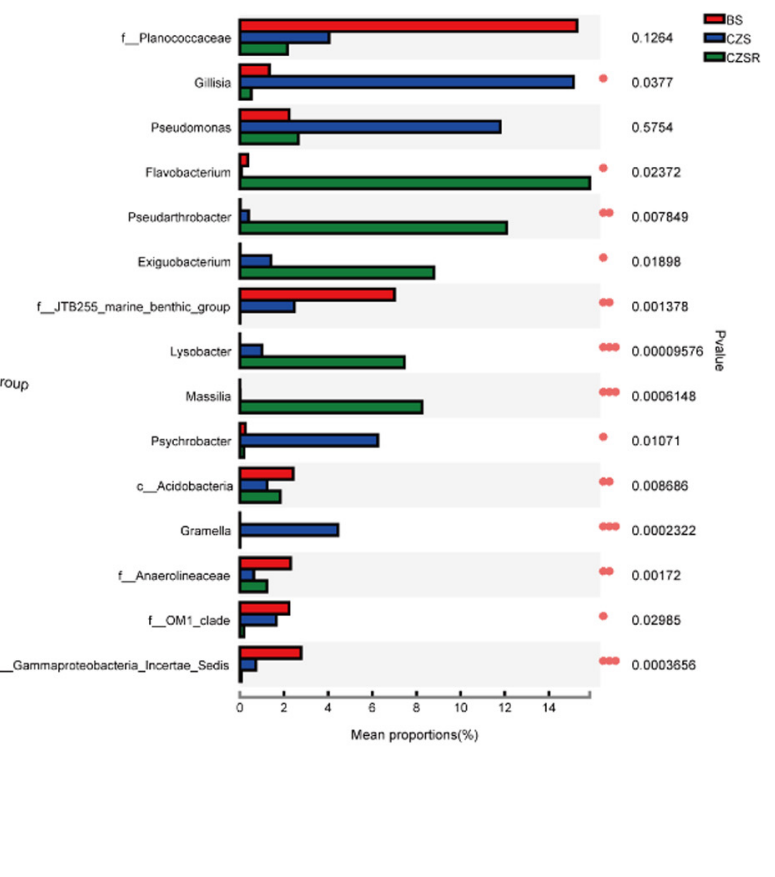

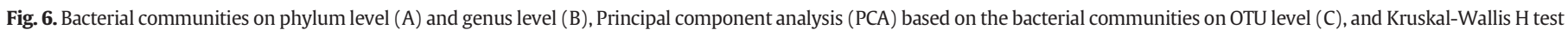
based on bacterial communities (D). BS, sediments in the Bohai Sea; CZS, sediments in coastal areas in summer; CZS-R, sediments in rivers. 
Pseudomonas, Psychrobacter, Flavobacterium, Gramella and Exiguobacterium were detected with higher relative abundances. However, the relative abundances of different bacteria varied significantly in different sediment samples. For instance, Gillisia were detected with higher relative abundances in BS07, BS18, CZS03, CZS04, CZS05, CZS06, CZS07 and CZS08, and Pseudomonas were detected with higher relative abundances in BS03, BS22, CZS02, CZS09 and CZS10. The genus of Paenisporosarcina was dominant in BS07, BS09 and BS17, the genus of Desulfobulbus was dominant in BS08, BS14 and BS25, and the genus of Planococcus was dominant in BS06 and BS20. In particular, the dominant genera of bacteria were distinctive in different coastal and river sediment samples, those were Lysobacter (CZS01), Flavobacterium (CZS06R), Exiguobacterium (CZS07R), Pseudarthrobacter (CZS08R) and Maritimimonas (CZS11). The complex environment and human activities contributed to the various bacterial communities in coastal areas.

Furthermore, PCA analysis was performed based on the bacterial communities on OTU level. As shown in Fig. 6C, the sediment samples collected from coastal areas were generally separated from the samples collected from the sea, which further confirmed that the bacterial communities differed from the sea to coastal areas. Fig. 6D described the variances between sediments collected from the sea (BS), the coast (CZS) and river (CZSR). The significant variances were assigned to the genera of Gillisia, Flavobacterium, Pseudarthrobacter, Exiguobacterium, Lysobacter, Massilia, Psychrobacter, Gramella, etc., revealing the representative bacterial communities in different sediment samples.

\subsection{Network analysis between ARGs and bacterial communities}

The correlations between target ARGs, intI1, hmt, 16S rRNA gene and bacterial communities on genus level (top 100) were visualized by the co-occurrence network (Fig. 7). A total of 768 connections with strong and significant correlations (Spearman's correlation coefficient $>0.6$, $\mathrm{p}<0.05$ ) were identified, which were significantly higher than those in water samples as previous research (Lu et al., 2019). Connections between gene \& gene, gene \& genus and genus \& genus accounted for $6.25 \%, 3.26 \%$ and $90.49 \%$, respectively.
There were close correlations between ARGs encoding resistance to aminoglycosides, $\beta$-lactams and quinolones (especially qnrS), which have been widely used to cure the human diseases. The qnrS was positively correlated with ten subtypes of ARGs, blaOXA30 was significantly correlated with seven ARG subtypes, and sul1, ermF, strA, blaTEM, blaCMY and $c p h A$ were positively related to six ARG subtypes. Interestingly, there was positive and significant correlation with the gene of $h m t$ and qnrS. Meanwhile, the connections between different bacterial genera were close and complex. There were more than thirty significant connections were identified between the genera of Haliea, Sva0081_sediment_group, Lutimonas, Lysobacter, Caldithrix, Arenibacter and other bacterial genera.

More importantly, the intI1 exhibited strong and significant correlations with 15 genera of bacteria, which were speculated to serve as the potential host bacteria for the horizontal transfer of ARGs. Target genes of intI1 and aadA2-03 were assigned to Lysobacter, Proteiniclasticum and Defluviimonas, and sul2 and aadA2-03 were assigned to the genus of Thiobacillus. Furthermore, connections between sul1 \& Carnobacterium, tetB \& Zeaxanthinibacter, qnrB \& Rubripirellula, qnrB \& Bythopirellula and aadA2-03 \& Rhodobacter were also determined. In particular, the genera mentioned above were generally detected with higher abundance in coastal areas, except that the genus of Rubripirellula was detected with high relative abundance in the sea and parts of coastal sampling sites. Interestingly, Carnobacterium was detected with higher abundance in the Laizhou Bay, for example, BS15, B17 and B18 in the sea, and CZO6 and CZO7 in coastal areas. Therefore, it could be speculated that the bacteria mentioned above might be the possible hosts of ARGs, which exhibited higher risks in coastal areas, especially in the Laizhou Bay areas. Coastal areas were hot spots of antibiotic resistance due to the intensive anthropogenic activities along the coastline of the Bohai Sea, the typical semi-enclosed sea.

\section{Conclusions}

In conclusion, a large-scale sampling was accomplished for investigating the occurrence and spatial distribution of ARGs in the typical semi-enclosed continental shelf sea and coastal areas. The total abundances of ARGs differed greatly in different sampling sites, while ARGs

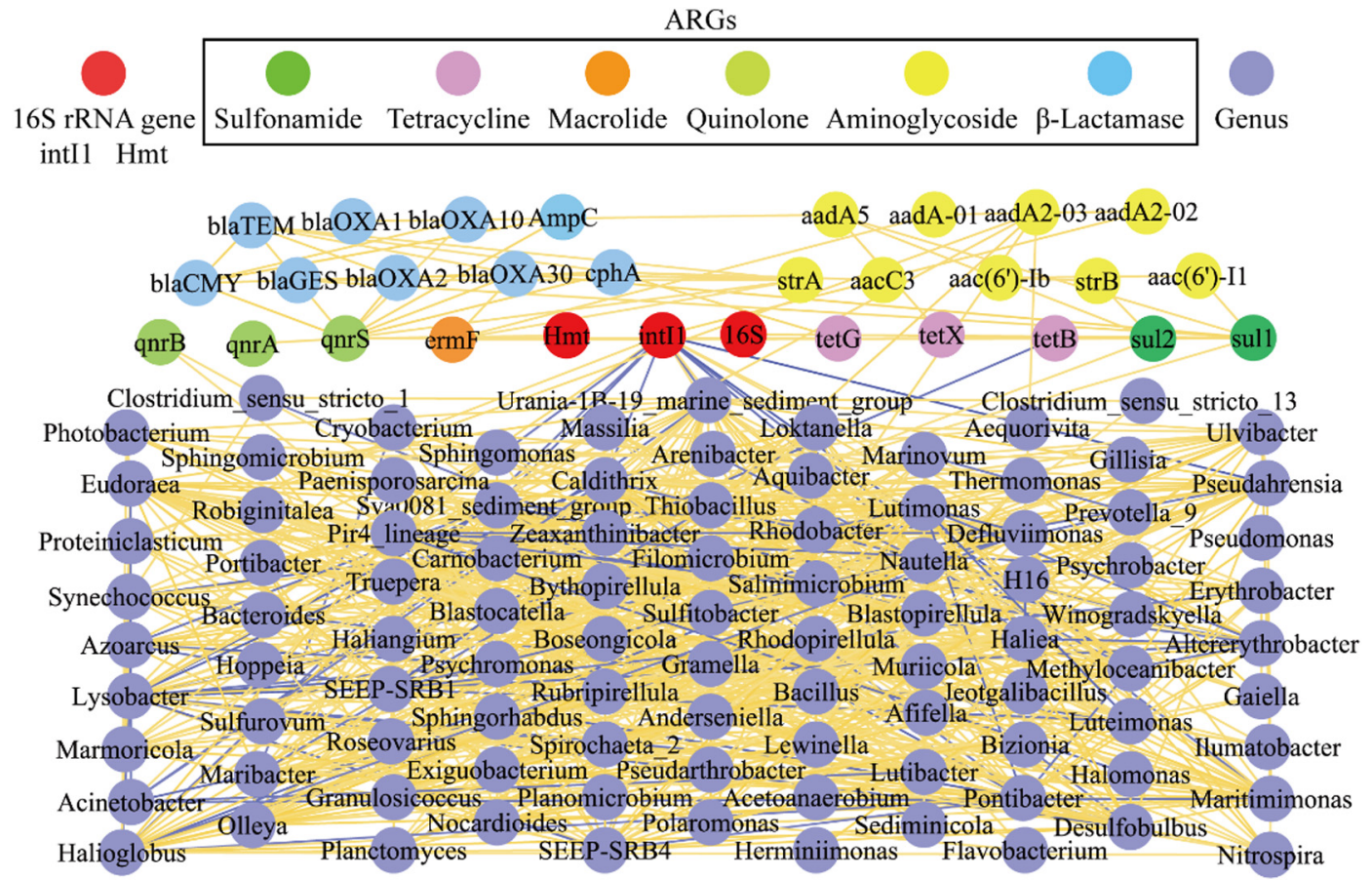

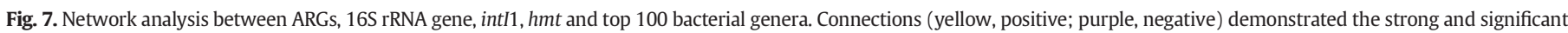
correlations (Spearman's correlation coefficient $>0.6, \mathrm{p}<0.05$ ) 
were more abundant in the Laizhou Bay, the south of Bohai Bay and the eastern of Bohai Central Sea. Sulfonamides resistance genes were ubiquitous and abundant, while $\beta$-lactams resistance genes hold lower abundances. The basic qualities of sediments played important roles on the distribution of ARGs by PCA and RDA analysis. Bacterial communities were identified and network analysis has been conducted, revealing the potential host bacteria of ARGs with higher relative abundances in coastal areas. This research provide a comprehensive investigations into the large-scale spatial distribution patterns of ARGs in sediments in the Bohai Sea, supplying basis data for further analysis and formulation of effective strategies to control the pollution of antibiotic resistance.

\section{CRediT authorship contribution statement}

Yuxuan Zhang: Investigation, Formal analysis, Data curation, Methodology, Methodology, Writing - original draft. Jian Lu: Conceptualization, Funding acquisition, Project administration, Supervision, Resources, Writing - review \& editing. Jun Wu: Methodology, Investigation, Software, Writing - review \& editing. Jianhua Wang: Formal analysis. Yichen Lin: Software.

\section{Declaration of competing interest}

The authors declare that they have no known competing financial interests or personal relationships that could have appeared to influence the work reported in this paper.

\section{Acknowledgements}

This work was supported by National Natural Science Foundation of China (41877131), Taishan Scholars Project of Shandong Province (No. tsqn201812116), One Hundred Talents Program of Chinese Academy of Sciences (Y629041021), Science and Technology Service Network Initiative of the Chinese Academy of Sciences (KFJ-STS-QYZX-114), TwoHundred Talents Plan of Yantai (Y739011021), and Youth Innovation Team Project for Talent Introduction and Cultivation in Universities of Shandong Province.

\section{Appendix A. Supplementary data}

Supplementary data to this article can be found online at https://doi. org/10.1016/j.scitotenv.2020.137712.

\section{References}

Chen, B.W., Liang, X.M., Huang, X.P., Zhang, T., Li, X.D., 2013a. Differentiating anthropogenic impacts on ARGs in the Pearl River estuary by using suitable gene indicators. Water Res. 47 (8), 2811-2820. https://doi.org/10.1016/j.watres.2013.02.042.

Chen, B.W. Yang Y, Liang X.M. Yu, K, Zhang T, Li, X.D, 2013b. Metagenomic profiles of antibiotic resistance genes (ARGs) between human impacted estuary and deep ocean sediments. Environ. Sci. Technol. 47 (22), 12753-12760. https://doi.org/10.1021/ es403818e.

Chen, B.W., Lin, L., Fang, L., Yang, Y., Chen, E.Z., Yuan, K., Zou, S.C., Wang, X.W., Luan, T.G. 2018. Complex pollution of antibiotic resistance genes due to beta-lactam and aminoglycoside use in aquaculture farming. Water Res. 134, 200-208. https://doi.org/ 10.1016/j.watres.2018.02.003.

Chen, J.Y., Su, Z.G., Dai, T.J., Huang, B., Mu, Q.L., Zhang, Y.M., 2019. Occurrence and distribution of antibiotic resistance genes in the sediments of the East China Sea bays. J. Environ. Sci. 81, 156-167. https://doi.org/10.1016/j.jes.2019.01.016.

Dang, B.J., Mao, D.Q., Xu, Y., Luo, Y., 2017. Conjugative multi-resistant plasmids in Haihe River and their impacts on the abundance and spatial distribution of antibiotic resistance genes. Water Res. 111, 81-91. https://doi.org/10.1016/j.watres.2016.12.046.

D’Costa, V.M., King, C.E., Kalan, L., Morar, M., Sung, W.W.L., Schwarz, C., et al., 2011. Antibiotic resistance is ancient. Nature 477, 457-461. https://doi.org/10.1038/ nature 10388
Duan, L.Q., Song, J.M., Xu, Y.Y., Li, X.G., Zhang, Y., 2010. The distribution, enrichment and source of potential harmful elements in surface sediments of Bohai Bay, North China. J. Hazard. Mater. 183, 155-164. https://doi.org/10.1016/j.jhazmat.2010.07.005.

Gillings, M.R., Gaze, W.H., Pruden, A., Smalla, K., Tiedje, J.M., Zhu, Y.G., 2015. Using the class 1 integron-integrase gene as a proxy for anthropogenic pollution. ISME J 9, 1269-1279. https://doi.org/10.1038/ismej.2014.226.

Jia, S.Y., Zhang, X.X., Miao, Y., Zhao, Y.T., Ye, L., Li, B., et al., 2017. Fate of antibiotic resistance genes and their associations with bacterial community in livestock breeding wastewater and its receiving river water. Water Res. 124, 259-268. https://doi.org/ 10.1016/j.watres.2017.07.061.

Jia, J., Guan, Y.J., Cheng, M.O., Chen, H., He, J.F., Wang, S., et al., 2018. Occurrence and distribution of antibiotics and antibiotic resistance genes in Ba River, China. Sci. Total Environ. 642, 1136-1144. https://doi.org/10.1016/j.scitotenv.2018.06.149.

Lu, X.M., Lu, P.Z., 2020. Seasonal variations in antibiotic resistance genes in estuarine sediments and the driving mechanisms. J. Hazard. Mater. 383, 121164. https://doi.org/ 10.1016/j.jhazmat.2019.121164.

Lu, J., Zhang, Y., Wu, J., Wang, J., Zhang, C., Lin, Y.C., 2019. Occurrence and spatial distribution of antibiotic resistance genes in the Bohai Sea and Yellow Sea areas, China. Environ. Pollut. 252, 450-460. https://doi.org/10.1016/j.envpol.2019.05.143.

Lu, J., Zhang, Y., Wu, J., 2020a. Continental-scale spatio-temporal distribution of antibiotic resistance genes in coastal waters along coastline of China. Chemosphere 247, 125908. https://doi.org/10.1016/j.chemosphere.2020.125908.

Lu, J., Zhang, Y., Wu, J., Wang, J., Cai, Y., 2020b. Fate of antibiotic resistance genes in reclaimed water reuse system with integrated membrane process. J. Hazard. Mater. 382, 121025. https://doi.org/10.1016/j.jhazmat.2019.121025.

Pei, R.T., Kim, S.C., Carlson, K.H., Pruden, A., 2006. Effect of river landscape on the sediment concentrations of antibiotics and corresponding antibiotic resistance genes (ARG). Water Res. 40, 2427-2435. https://doi.org/10.1016/j.watres.2006.04.017.

Peng, F., Isabwe, A., Guo, Y.Y., Chen, H.H., Yang, J., 2019. An extensively shared antibiotic resistome among four seasons suggests management prioritization in a subtropical riverine ecosystem. Sci. Total Environ. 673, 533-540. https://doi.org/10.1016/j. scitotenv.2019.04.031.

Šmilauer, P., Lepš, J., 2014. Multivariate Analysis of Ecological Data Using CANOCO 5. Cambridge University Press, Cambridge.

Song, J.M., Duan, L.Q., 2019. Chapter 17 - the Bohai Sea. In: Sheppard, C. (Ed.), World Seas: An Environmental Evaluation, Second edition Academic Press, pp. 377-394. https:// doi.org/10.1016/B978-0-08-100853-9.00024-5.

Tan, L., Li, L.Y., Ashbolt, N., Wang, X.L., Cui, Y.X., Zhu, X., et al., 2018. Arctic antibiotic resistance gene contamination, a result of anthropogenic activities and natural origin. Sci. Total Environ. 621, 1176-1184. https://doi.org/10.1016/j.scitotenv.2017.10.110.

Wang, F.H., Qiao, M., Lv, Z.E., Guo, G.X., Jia, Y., Su, Y.H., et al., 2014. Impact of reclaimed water irrigation on antibiotic resistance in public parks, Beijing, China. Environ. Pollut. 184, 247-253. https://doi.org/10.1016/j.envpol.2013.08.038.

Wang, J., Lu, J., Zhang, Y., Wu, J., Luo, Y., Liu, H., 2018. Metagenomic analysis of antibiotic resistance genes in coastal industrial mariculture systems. Bioresour. Technol. 253, 235-243. https://doi.org/10.1016/j.biortech.2018.01.035.

Yang, Y.Y., Li, Z., Song, W.J., Du, L.N., Ye, C., Zhao, B., et al., 2019a. Metagenomic insights into the abundance and composition of resistance genes in aquatic environments: influence of stratification and geography. Environ. Int. 127, 371-380. https://doi.org/ 10.1016/j.envint.2019.03.062.

Yang, Y.Y., Liu, G.H., Song, W.J., Ye, C., Lin, H., Li, Z., et al., 2019b. Plastics in the marine environment are reservoirs for antibiotic and metal resistance genes. Environ. Int. 123, 79-86. https://doi.org/10.1016/j.envint.2018.11.061.

Yin, X.L., Deng, Y., Ma, L.P., Wang, Y.L., Chan, L.Y.L., Zhang, T., 2019. Exploration of the antibiotic resistome in a wastewater treatment plant by a nine-year longitudinal metagenomic study. Environ. Int. 133, 105270. https://doi.org/10.1016/j. envint.2019.105270.

Yuan, K., Wang, X.W., Chen, X., Zhao, Z.Q., Fang, L., Chen, B.Y., et al., 2019. Occurrence of antibiotic resistance genes in extracellular and intracellular DNA from sediments collected from two types of aquaculture farms. Chemosphere 234, 520-527. https://doi. org/10.1016/j.chemosphere.2019.06.085.

Zhang, Q.Q., Ying, G.G., Pan, C.G., Liu, Y.S., Zhao, J.L., 2015. Comprehensive evaluation of antibiotics emission and fate in the river basins of China: source analysis, multimedia modeling, and linkage to bacterial resistance. Environ. Sci. Technol. 49, 6772-6782. https://doi.org/10.1021/acs.est.5b00729.

Zhang, Y.P., Niu, Z.G., Zhang, Y., Zhang, K., 2018. Occurrence of intracellular and extracellular antibiotic resistance genes in coastal areas of Bohai Bay (China) and the factors affecting them. Environ. Pollut. 236, 126-136. https://doi.org/10.1016/j. envpol.2018.01.033.

Zhao, R.X., Feng, J., Yin, X.L., Liu, J., Fu, W.J., Berendonk, T.U., et al., 2018. Antibiotic resistome in landfill leachate from different cities of China deciphered by metagenomic analysis. Water Res.Water Research 134, 126-139. https://doi.org/ 10.1016/j.watres.2018.01.063.

Zhu, Y.G., Zhao, Y., Li, B., Huang, C.L., Zhang, S.Y., Yu, S., et al., 2017. Continental-scale pollution of estuaries with antibiotic resistance genes. Nat. Microbiol. 2, 16270. https:// doi.org/10.1038/nmicrobiol.2016.270. 